\title{
Introduzione alla conoscenza della sottofamiglia Aleocharinae della Guyana Francese: Parte III ${ }^{1}$ (Coleoptera, Staphylinidae)
}

\author{
Con 87 figure \\ ROBERTO PACE ${ }^{2}$ \\ ${ }^{1} 286^{\circ}$ "Contributo alla conoscenza delle Aleocharinae". \\ ${ }^{2}$ Via Vittorio Veneto, 13, 37032 Monteforte d’Alpone (Verona), Italia. - pace.ent@tiscali.it \\ Published on 2014-07-31
}

\section{Summary}

Description of 23 new species of the tribe Athetini: Ischnopoda pulchritudinis n. sp., Xyronarchusa amazonica n. gen., n. sp., Atheta (Acrotona) obscurepyga n. sp., Atheta (Acrotona) tanpokensis n. sp., Atheta (Acrotona) guyterminicornis n. sp., Atheta (Acrotona) subparcior n. sp., Atheta (Acrotona) guyminima n. sp., Atheta (Microdota) ungulifera n. sp., Atheta (Microdota) guyarufa n. sp., Atheta (Xestota) guyattenuata n. sp., Atheta (Datomicra) guymimetica n. sp., Atheta (Datomicra) guydivergens n. sp., Atheta (Datomicra) guyanelegans n. sp., Atheta (Datomicra) guydifferens n. sp., Atheta (Datomicra) caussadensis n. sp., Atheta (Datomicra) camopiensis n. sp., Atheta (Datomicra) struyvei n. sp., Atheta (Gyroatheta subg. n.) guyanicola n. sp., Lamprostiba pulchra n. sp., Parademosoma (Elixusa subg. n.) guyanensis n. sp., Dolerella cayennensis n. sp., Dinusella angularis n. sp., Dinusella pulchripennis n. sp. Additional distributional records are reported for 7 species. Two new genera Xyronarchusa n. gen. and Dolerella n. gen. and two new subgenera Gyroatheta subg. n. of Atheta and Elixusa subg. n. of Parademosoma are described. All new species are illustrated and compared with similar species.

\section{Key words}

Coleoptera, Staphylinidae, Aleocharinae, taxonomy, French Guiana.

\section{Zusammenfassung}

23 neue Arten der Tribus Athetini werden beschrieben: Ischnopoda pulchritudinis n. sp., Xyronarchusa amazonica n. gen., n. sp., Atheta (Acrotona) obscurepyga n. sp., Atheta (Acrotona) tanpokensis n. sp., Atheta (Acrotona) guyterminicornis n. sp., Atheta (Acrotona) subparcior n. sp., Atheta (Acrotona) guyminima n. sp., Atheta (Microdota) ungulifera n. sp., Atheta (Microdota) guyarufa n. sp., Atheta (Xestota) guyattenuata n. sp., Atheta (Datomicra) guymimetica n. sp., Atheta (Datomicra) guydivergens n. sp., Atheta (Datomicra) guyanelegans n. sp., Atheta (Datomicra) guydifferens n. sp., Atheta (Datomicra) caussadensis n. sp., Atheta (Datomicra) camopiensis n. sp., Atheta (Datomicra) struyvei n. sp., Atheta (Gyroatheta subg. n.) guyanicola n. sp., Lamprostiba pulchra n. sp., Parademosoma (Elixusa subg. n.) guyanensis n. sp., Dolerella cayennensis n. sp., Dinusella angularis n. sp., Dinusella pulchripennis n. sp. Für 7 Arten werden zusätzliche Daten aufgeführt. Zwei neue Gattungen, Xyronarchusa n. gen. und Dolerella n. gen. und zwei neue Untergattungen Gyroatheta subg. n. von Atheta und Elixusa subg. n. von Parademosoma werden beschrieben. Jede neue Art wird illustriert und mit ähnlichen Arten verglichen. 


\section{Riassunto}

Descrizione di 23 nuove specie della tribù Athetini: Ischnopoda pulchritudinis n. sp., Xyronarchusa amazonica n. gen., n. sp., Atheta (Acrotona) obscurepyga n. sp., Atheta (Acrotona) tanpokensis n. sp., Atheta (Acrotona) guyterminicornis n. sp., Atheta (Acrotona) subparcior n. sp., Atheta (Acrotona) guyminima n. sp., Atheta (Microdota) ungulifera n. sp., Atheta (Microdota) guyarufa n. sp., Atheta (Xestota) guyattenuata n. sp., Atheta (Datomicra) guymimetica n. sp., Atheta (Datomicra) guydivergens n. sp., Atheta (Datomicra) guyanelegans n. sp., Atheta (Datomicra) guydifferens n. sp., Atheta (Datomicra) caussadensis n. sp., Atheta (Datomicra) camopiensis n. sp., Atheta (Datomicra) struyvei n. sp., Atheta (Gyroatheta subg. n.) guyanicola n. sp., Lamprostiba pulchra n. sp., Parademosoma (Elixusa subg. n.) guyanensis n. sp., Dolerella cayennensis n. sp., Dinusella angularis n. sp., Dinusella pulchripennis n. sp. Sono riportati dati distributivi supplementari per 7 specie. Sono descritti due nuovi generi Xyronarchusa n. gen. e Dolerella n. gen. e due nuovi sottogeneri Gyroatheta subg. n. di Atheta ed Elixusa subg. n. di Parademosoma. Ogni specie nuova è illustrata e comparata con specie simili.

\section{Acronimi}

Gli holotypi e paratypi delle nuove specie e altro materiale sono conservati nei seguenti istituti:

FMNHC: Field Museum of Natural History, Chicago

IRSNB: Institut royal des Sciences naturelles de Belgique, Bruxelles

MNHUB: Museum für Naturkunde der HumboldtUniversität, Berlin

SDEI: Senckenberg Deutsches Entomologisches Institut, Müncheberg

\section{ATHETINI}

\section{Ischnopoda pulchritudinis n. sp.}

(Figg. 1 e 24-25)

Materiale tipico: Holotype $\sigma^{\star}$, Guyane Fr., Saramaka, 13.XI.2011, leg. T. Struyve (IRSNB).

Descrizione: Lunghezza $2,7 \mathrm{~mm}$. Corpo lucido e rossiccio, uroterghi liberi basali primo, secondo e base del terzo giallo-rossicci, restante addome nero, antenne brune con i tre antennomeri basali giallo-rossiccio e undicesimo giallo pallido. Occhi più corti della regione postoculare, in visione dorsale. Secondo antennomero più corto del primo, terzo più corto del secondo, quarto a decimo più lunghi che larghi. Reticolazione del capo molto superficiale, quella del pronoto forte nel fondo dell'ampia cavità mediana, ai lati la reticolazione è molto evanescente. Reticolazione di elitre e addome non visibile. Punteggiatura del capo non visibile. Granulosità del pronoto molto superficiale, quella delle elitre fine e molto superficiale, quella dell'addome ridotta a sparsi granuli. Edeago fig. 24-25.

Comparazioni: La nuova specie, oltre che per il colore del corpo, è distinta da I. mexicana (BERNHAUER, 1929) del Messico, per la lunghezza dell'undicesimo antennomero, lungo quanto i 4 precedenti antennomeri riuniti, mentre in mexicana l'undicesimo antennomero è lungo quanto i 2 predenti antennomeri riuniti. I. gnypetiformis (Bernhauer, 1934) dell'Argentina ha l'undicesimo antennomero lungo quanto i 3 precedenti antennomeri riuniti. Edeago figg. 24-25.

Etimologia: La nuova specie ha nome che significa "della bellezza”. La bellezza è quella del corpo della nuova specie, per la sua colorazione.

\section{Gnypeta pannosa PACE, 1996}

Gnypeta pannosa PACE, 1996: 644

$10^{\star}$ e 2 우 우, Guyane Fr., Caussade, 7.XI.2011, leg. T. Struyve (IRSNB, SDEI).

Distribuzione: Colombia.

\section{Xyronarchusa n. gen.}

(Figg. 2 e 26-29)

Diagnosi: Habitus simile ad Atheta Thomson, 1858 del sottogenere Acrotona Thomson, 1858, ma la ligula e la forma della spermateca sono molto differenti. La ligula del nuovo genere è intera e a forma di mezza luna a base ristretta, fig. 27, e non divisa in due rami a forma di Y come in Acrotona e Atheta (Atheta). La spermateca del nuovo genere, fig. 26, è composta di numerose spire ampie e strette. In Acrotona le spire prossimali della spermateca sono da una a tre e ampie. Il nuovo genere si presenta come un intermedio tra Placusa ERICHson, 1837, dei Placusini, che ha ligula intera, formula tarsale 4-4-5 e palpi labiali di 2 articoli, e Atheta che ha formula tarsale 4-5-5 e palpi labiali di tre articoli, fig. 27. La forma della ligula del nuovo genere ricorda quello degli Athetini Codoglossa SawADA, 1980 dell'Indonesia, ma nel nuovo genere essa è molto ristretta alla base e mancano due setole apicali. La spermateca del nuovo genere ha forma completamente differente da quella di 
Codoglossa che è semplice ovvero non avvolta in spire. L'habitus del nuovo genere è simile a Acrotona, quello di Codoglossa a Microdota Mulsant \& Rey, 1873. Maxilla con palpo mascellare fig. 28, mento fig. 29.

Tipo del genere: Xyronarchusa amazonica, n. sp.

Etimologia: Il nome del nuovo genere significa "Che è un rasoio antico" per la forma della ligula simile a un rasoio greco-romano antico, dal greco antico $\xi u \rho o ́ v=$ rasoio, ápxaîo $\varsigma=$ antico e ov́ $\sigma \alpha=$ che è.

\section{Xyronarchusa amazonica n. sp.}

(Figg. 2 e 26-29)

Materiale tipico: Holotype + , Guyane Fr., Caussade, 7.XI.2011, leg. T. Struyve (SDEI).

Descrizione: Lunghezza $2 \mathrm{~mm}$. Corpo lucido e nerobruno, pronoto ed elitre giallo-bruni, antenne nere con i due antennomeri basali giallo-rossicci, zampe gialle. Occhi più lunghi della regione postoculare, in visione dorsale. Secondo antennomero lungo quanto il primo, terzo più corto del secondo, quarto a decimo trasversi. Reticolazione del capo evidente, quella di pronoto, elitre e addome superficiale, sugli uroterghi liberi quarto e quinto molto trasversa. Punteggiatura del capo fine, fitta e superficiale. Granulosità di pronoto ed elitre fitta ed evidente. Spermateca fig. 26.

Etimologia: La nuova specie prende nome dalla giungla dell'Amazzonia.

\section{Atheta (Acrotona) muellerina PACE, 1996}

Atheta (Acrotona) muellerina PACE, 1996: 648

15 esemplari, Guyane Fr., Caussade, 7.XI.2011, leg. T. Struyve; 5 esemplari, Guyane Fr., Barrage Petit Saut, 10.XI.2011, leg. T. Struyve (IRSNB, SDEI).

Distribuzione: Colombia.

\section{Atheta (Acrotona) obscurepyga n. sp.}

(Figg. 3 e 30-32)

Materiale tipico: Holotype $\sigma^{\star}$, Guyane Fr., Caussade, 7.XI.2011, leg. T. Struyve (SDEI).

Paratypes: 10 esemplari, stessa provenienza; 1 \%, Guyane Fr., Tonate, 6.XI.2011, leg. T. Struyve (IRSNB, SDEI).

Descrizione: Lunghezza 1,8 $\mathrm{mm}$. Corpo lucido e rossiccio, capo bruno, elitre bruno-rossicce, urotergo libero quarto e pigidio bruni, quinto urotergo libero giallo-rossiccio, antenne brune con i due antennomeri basali giallo-rossicci, zampe giallo-rossicce. Occhi lunghi quanto la regione postoculare, in visione dorsale. Secondo antennomero lungo quanto il primo, terzo più corto del secondo, quarto lungo quanto largo, quinto a decimo trasversi. Reticolazione di capo, elitre e quinto urotergo libero superficiale, quella di pronoto e resto dell'addome non visibile. Granulosità del capo poco evidente, quella di pronoto, elitre e addome fitta, fine ed evanescente. Edeago figg. 30-31, spermateca fig. 32.

Comparazioni: Per il colore del corpo la nuova specie è simile ad A. flavonigra Bernhauer, 1908 del Paraguay, di cui ho esaminato 2 ㅇ 우 della serie tipica (FMNHC). Se ne distingue per il colore giallo-rossiccio del pronoto, in flavonigra bruno-rossiccio, per la parte prossimale della spermateca attenuata e non larga come in flavonigra e per l'introflessione apicale del bulbo distale della spermateca con base molto larga, mentre è stretta in flavonigra.

Etimologia: La nuova specie prende nome dal suo peculiare carattere: il pigidio oscuro. Solitamente il pigidio delle Aleocharinae è giallo-rossiccio se il corpo non è nero o è nero-bruno.

\section{Atheta (Acrotona) tanpokensis n. sp. \\ (Figg. 4 e 33-35}

Materiale tipico: Holotype $\sigma^{\star}$, Guyane Fr., Coralie, 15.XI.2011, leg. T. Struyve (IRSNB).

Paratypus: 1 \%, Guyane Fr., Coralie, 16.XI.2011, leg. T. Struyve (SDEI).

Descrizione: Lunghezza $2 \mathrm{~mm}$. Corpo lucido e bruno, pigidio giallo-rossiccio, antenne brune con i due antennomeri basali giallo-bruni, zampe gialle. Occhi più lunghi della regione postoculare, in visione dorsale. Secondo antennomero più corto del primo, terzo più corto del secondo, quarto lungo quanto largo, quinto a decimo trasversi. Reticolazione di capo ed elitre superficiale, quella di pronoto e addome invisibile. Granulosità del capo fitta e poco evidente, quella di pronoto ed elitre fitta e saliente, quella dell'addome fitta e fine. Edeago figg. 33-34, spermateca fig. 35.

Comparazioni: Non sono note specie del sottogenere Acrotona con un lungo flagello interno dell'edeago.

Etimologia: La nuova specie deriva il suo nome dal fiume Tanpok della Guyana Francese. 


\section{Atheta (Acrotona) guyterminicornis n. sp.}

(Figg. 5 e 36-37)

Materiale tipico: Holotype $\sigma^{\star}$, Guyane Fr., Saramaka, 13.XI.2011, leg. T. Struyve (SDEI).

Descrizione: Lunghezza 1,8 mm. Corpo lucido e nerobruno, pigidio rossiccio, antenne brune con undicesimo antennomero e zampe rossicci. Occhi più lunghi della regione postoculare, in visione dorsale. Secondo antennomero più corto del primo, terzo più corto del secondo, quarto a decimo trasversi. Reticolazione dell'avancorpo invisibile, quella dell'addome trasversa e molto superficiale. Granulosità dell'avancorpo fine, fitta e molto saliente. Edeago figg. 36-37.

Comparazioni: Ledeago della nuova specie è simile a quello di A. parcior Bernhauer, 1927 dell'Argentina, di cui ho esaminato l'olotipo $\sigma^{*}$ (FMNHC). Se ne distingue per l'undicesimo antennomero rossiccio e non bruno come in parcior, per l'edeago meno lungo e con una spina interna corta, mentre è lunga in parcior.

Etimologia: La nuova specie ha nome che richiama l'attenzione sullestremità rossiccia delle antenne.

\section{Atheta (Acrotona) subparcior n. sp.}

(Figg. 6 e 38)

Materiale tipico: Holotype + , Guyane Fr., Barrage Petit Saut, 10.XI.2011, leg. T. Struyve (IRSNB).

Descrizione: Lunghezza 1,9 mm. Corpo lucido e nero pece, pigidio giallo-rossiccio, antenne nere, zampe rossicce. Occhi più lunghi della regione postoculare, in visione dorsale. Secondo antennomero lungo quanto il primo, terzo lungo quanto il secondo, quarto a decimo trasversi. Reticolazione del corpo invisibile, ma sul quinto urotergo libero molto superficiale. Granulosità del corpo fitta e saliente. Spermateca fig. 38 .

Comparazioni: La nuova specie ha spermateca simile a quella di $A$. parcior BernHAUer, 1927 dell'Argentina, di cui ho esaminato l'olotipo $\sigma^{\star}$ e una serie di esemplari $\mathrm{o}^{\star} \mathrm{o}^{\boldsymbol{x}} \mathrm{e}$ 우 우 (FMNHC), ma la sua lunghezza è minore e l'introflessione apicale del bulbo distale è stretta e non larga come in parcior. Il bulbo basale della spermateca della nuova specie è largo, quello di parcior stretto.

Etimologia: La nuova specie ha nome che indica la sua affinità con $A$. parcior.

\section{Atheta (Acrotona) guyminima n. sp. \\ (Figg. 7 e 39-41)}

Materiale tipico: Holotype + , Guyane Fr., Caussade, 6.XI.2011, leg. T. Struyve (SDEI).

Paratypes: 4 우, stessa provenienza (IRSNB, SDEI).

Descrizione: Lunghezza $1,5 \mathrm{~mm}$. Corpo lucido e nero pece, antenne nere, zampe giallo-brune. Occhi più corti della regione postoculare, in visione dorsale. Secondo antennomero lungo quanto il primo, terzo più corto del secondo, quarto lungo quanto largo, quinto a decimo trasversi. Reticolazione del corpo non visibile. Granulosità dell'avancorpo fitta, fine e saliente, quella dell'addome fitta, fine e superficiale. Edeago figg. 39-40, spermateca fig. 41 .

Comparazioni: Lampia spira prossimale della spermateca della nuova specie è simile a quella di A. neasuspiciosa PACE, 2008 dell'Ecuador. La spermateca della nuova specie è lunga $0,16 \mathrm{~mm}$, quella di neasuspiciosa $0,27 \mathrm{~mm}$. L'introflessione apicale del bulbo distale della spermateca della nuova specie è profonda, quella di neasuspiciosa molto corta.

Etimologia: La nuova specie ha nome che significa "molto piccola della Guyana Francese" a motivo della taglia corporea di 1,5 $\mathrm{mm}$.

\section{Atheta (Microdota) brasiliana Bernhauer, 1908}

Atheta (Microdota) brasiliana BERNHAUer, 1908: 358

6 esemplari, Guyane Fr., Caussade, 7.XI.2011, leg. T. Struyve (IRSNB, SDEI).

Distribuzione: Brasile.

Nota: Da me esaminata la serie tipica di questa specie (FMNHC).

\section{Atheta (Microdota) ungulifera n. sp. \\ (Figg. 8 e 42-43)}

Materiale tipico: Holotype $\sigma^{*}$, Guyane Fr., Coralie, 16.XI.2011, leg. T. Struyve (IRSNB).

Descrizione: Lunghezza $2 \mathrm{~mm}$. Corpo lucido e giallo-rossiccio, elitre e quarto urotergo libero bruno-rossicci, antenne brune con i due antennomeri basali, base del terzo e undicesimo giallo-rossicci. zampe giallo-rossicce. Occhi lunghi quanto la regione posto- 
culare, in visione dorsale. Secondo antennomero lungo quanto il primo, terzo più corto del secondo, quarto a decimo trasversi. Reticolazione del capo evidente, quella del pronoto superficiale, quella delle elitre molto evanescente, quella dell'addome non visibile. Punteggiatura di capo e pronoto fitti e superficiale. Granulosità delle elitre poco saliente, quella dell'addome fitta, ma sul quinto urotergo libero del maschio sparsa. Edeago figg. 42-43.

Comparazioni: I due pezzi copulatori interni ricurvi dell'edeago della nuova specie sono presenti anche nell'edeago di A. sufflavicollis PACE, 1990 del Brasile. Nella nuova specie l'edeago è stretto, in visione ventrale, e l'undicesimo antennomero è giallo-rossiccio, in sufflavicollis l'edeago è largo e l'undicesimo antennomero bruno.

Etimologia: La nuova specie ha nome che significa "portatrice di unghie" perché i due pezzi copulatori apicali interni e ricurvi dell'edeago hanno forma di unghie di zampa di Insetto.

\section{Atheta (Microdota) guyarufa n. sp}

(Figg. 9 e 44-45)

Materiale tipico: Holotype $\sigma^{x}$, Guyane Fr., Coralie, 16.XI.2011, leg. T. Struyve (SDEI).

Paratype: $1 \sigma^{\top}$, stessa provenienza (IRSNB).

Descrizione: Lunghezza 1,9 $\mathrm{mm}$. Corpo lucido e rossiccio, antenne rossicce con i due antennomeri basali e l'undicesimo giallo-rossicci, zampe giallo-rossicce. Occhi lunghi quanto la regione postoculare, in visione dorsale. Secondo antennomero più corto del primo, terzo più corto del secondo, quarto a decimo trasversi. Reticolazione del capo evidente, quella del pronoto invisibile, quella delle elitre superficiale, quella delladdome molto trasversa ed evanescente. Punteggiatura di capo e pronoto fitta ed evidente. Granulosità di elitre e addome fitta ed evidente. Pronoto con impressione discale superficiale. Edeago figg. 44-45.

Comparazioni: L'habitus della nuova specie è simile a quello di A. subida (Erichson, 1840) del Brasile di cui ho esaminato la serie tipica di $20^{*} o^{*}$ e 2 우, $n^{\circ} 5473$ (MNHUB). La nuova specie se ne distingue per l'edeago ampiamente arcuato al lato ventrale, mentre in subida non è arcuato. In visione ventrale, l'edeago della nuova specie è più largo di quello di subida.

Etimologia: La nuova specie prende nome dal colore del suo corpo e dalla Guyana Francese.

\section{Atheta (Xestota) guyattenuata n. sp.}

(Figg. 10 e 46-49)

Materiale tipico: Holotype $\sigma^{\star}$, Guyane Fr., Coralie, 16.XI.2011, leg. T. Struyve (IRSNB).

Paratypes: $30^{\top} o^{\star}$ e $5+9$, stessa provenienza (IRSNB, SDEI).

Descrizione: Lunghezza 1,6 mm. Corpo lucido e giallo-rossiccio, capo giallo-bruno, elitre, tranne la base, brune, metà posteriore degli uroterghi liberi terzo e quarto bruni, antenne nere con i due antennomeri basali gialli, zampe gialle. Occhi più lunghi della regione postoculare, in visione dorsale. Secondo antennomero più corto del primo, terzo più corto del secondo, quarto a decimo trasversi. Reticolazione di capo ed elitre superficiale, quella di pronoto e addome invisibile. Punteggiatura del corpo fitta ed evidente, assente sulla fascia longitudinale mediana e in avanti del capo. Edeago figg. 46-47, spermateca fig. 48, sesto urotergo libero del maschio fig. 49.

Comparazioni: Per la forma della spermateca, la nuova specie è affine a A. seminigra (ERICHSON, 1840) della Colombia, di cui ho esaminato l'olotipo $\circ$ (MNHUB). La spermateca della nuova specie è lunga $0,13 \mathrm{~mm}$, quella di seminigra $0,2 \mathrm{~mm}$. L'introflessione apicale del bulbo distale della spermateca della nuova specie è triangolare, fig. 48, di forma irregolare in seminigra. La lunghezza della spermateca della nuova specie, $0,11 \mathrm{~mm}$, è simile a quella di A.callicornis PACE, 1996 dell'Ecuador, ma questa specie ha il bulbo distale della spermateca stretto e la sua parte prossimale più lunga, $0,03 \mathrm{~mm}$, di quella della nuova specie, $0,02 \mathrm{~mm}$.

Etimologia: La nuova specie prende nome dalla Guyana Francese e dal carattere della parte prossimale della spermateca, che è attenuata in larghezza.

\section{Atheta (Datomicra) conformis (ERICHSON, 1840)}

Homalota conformis ERICHSON, 1839: 108

Atheta (s. str.) conformis; BeRNHAUER \& SCHEERPELTZ, 1926: 640 Atheta (Datomicra) conformis; PACE, 2008: 403

19 esemplari, Guyane Fr., Caussade, 6.XI.2011, leg. T. Struyve; 1 o e 1 오, Guyane Fr., Coralie, 15.XI.2011, leg. T. Struyve; 24 esemplari, Guyane Fr., Coralie, 16.XI.2011, leg. T. Struyve; $2 o^{\top} o^{*}$ e 1 \%, Guyane Fr., Saramaka, 13.XI.2011, leg. T. Struyve (IRSNB, SDEI).

Distribuzione: Colombia, Venezuela, Ecuador, Antille, Argentina, Brasile e Suriname.

Nota: Serie tipica da me esaminata (MNHUB). 


\section{Atheta (Datomicra) dichroa GravenHORST, 1802}

Aleochara dichroa GRAVENHORST, 1802: 186

Homalota dichroa; ERICHSON, 1840: 186

Atheta dichroa; PACE, 1987: 192

1 ㅇ, Guyane Fr., Barrage Petit Saut, 10.XI.2011, leg. T. Struyve (IRSNB).

Distribuzione: N America, Antille.

Nota: Serie tipica da me esaminata (MNHUB).

\section{Atheta (Datomicra) lurida (ERICHSON, 1840)}

Homalota lurida ERICHSON, 1839: 108

Atheta (Atheta) lurida; Bernhauer \& SCHEerpeltz, 1926: 645

Atheta (Datomicra) lurida; PACE, 2008: 404

2 o $^{\star}$ e 3 우 오, Guyane Fr., Caussade, 7.XI.2011, leg. T. Struyve; $10^{\star}$ Guyane Fr., Caussade, 6.XI.2011, leg. T. Struyve; 21 esemplari, Guyane Fr., Saramaka, 13.XI.2011, leg. T. Struyve (IRSNB, SDEI).

Distribuzione: Brasile.

Nota: Serie tipica da me esaminata (MNHUB).

\section{Atheta (Datomicra) pseudogagatina BernHAUER, 1908}

Atheta (s. str.) pseudogagatina BERNHAUER, 1934: 504

Atheta (Datomicra) pseudogagatina; PACE, 2008: 403

$2 o^{\star} o^{*}$ e 1 ㅇ, Guyane Fr., Caussade, 6.XI.2011, leg. T. Struyve (IRSNB, SDEI).

Distribuzione: Argentina, Brasile e Colombia.

Nota: Serie tipica da me esaminata (FMNHC).

\section{Atheta (Datomicra) guymimetica n. sp.}

(Figg. 11 e 50-53)

Materiale tipico: Holotype ${ }^{\star}$, Guyane Fr., Coralie, 16.XI.2011, leg. T. Struyve (SDEI).

Paratypes: $20^{\star} o^{*}$ e 2 우, stessa provenienza (IRSNB, SDEI).

Descrizione: Lunghezza 1,9 mm. Corpo lucido e nero-bruno, pronoto e base delle elitre rossicci, base dell'addome e pigidio giallo-rossicci, antenne brune con i due antennomeri basali e l'undicesimo giallo-rossicci, zampe giallo-rossiccio. Occhi lunghi quanto la regione postoculare, in visione dorsale. Secondo antennomero lungo quanto il primo, terzo lungo quanto il secondo, quarto a decimo trasversi. Reticolazione di capo ed elitre evidente, quella del pronoto molto superficiale, quella dell'addome molto trasversa e molto evanescente. Punteggiatura del capo fitta, fine e superficiale. Granulosità di pronoto ed elitre fine e fitta, quella del quinto urotergo libero meno fitta di quella dei restanti uroterghi liberi. Edeago figg. 50-51, spermateca fig. 52, sesto urotergo libero del maschio fig. 53.

Comparazioni: Per la forma della spermateca e il colore dell'undicesimo antennomero, la nuova specie è comparabile con A. conformis (ERICHSON, 1840) della Colombia, di cui ho esaminato la serie tipica (MNHUB). Tra i lobi del margine posteriore del sesto urotergo libero del maschio della nuova specie il bordo è seghettato, mentre in conformis non lo è. La base dell'addome della nuova specie è giallo-rossiccio, quello di conformis bruno. Ledeago della nuova specie è lungo $0,22 \mathrm{~mm}$, quello di conformis $0,36 \mathrm{~mm}$.

Etimologia: La nuova specie prende nome di mimetica per la forma delledeago che sembra quello di Atheta conformis, perciò per la somiglianza edeagica è facilmente confondibile con conformis. Altri caratteri aiutano a distinguerla da essa. Prende nome anche dalla Guyana Francese.

\section{Atheta (Datomicra) guydivergens n. sp. (Figg. 12 e 54-57)}

Materiale tipico: Holotype $\sigma^{x}$, Guyane Fr., Caussade, 6.XI.2011, leg. T. Struyve (IRSNB).

\section{Paratype: $1+$, stessa provenienza (SDEI).}

Descrizione: Lunghezza 2,1 mm. Corpo lucido e bruno, capo e pronoto neri, antenne nere con i due antennomeri basali bruni, zampe giallo-rossicce. Occhi più lunghi della regione postoculare, in visione dorsale. Secondo antennomero più corto del primo, terzo più corto del secondo, quarto a decimo trasversi. Reticolazione del capo evidente, quella del pronoto invisibile, quella delle elitre superficiale, quella dell'addome molto trasversa ed evidente. Punteggiatura di capo e pronoto evidente, assente su un'area triangolare frontale. Granulosità delle elitre fitta e superficiale, quella dell'addome fitta, ma sul quinto urotergo libero sparsa. Edeago figg. 54-55, spermateca fig. 56, sesto urotergo libero del maschio fig. 57.

Comparazioni: La spermateca della nuova specie è simile a quella di A.pseudogagatina Bernhauer, 1908 del Brasile, ma l'introflessione apicale del bulbo distale della stessa spermateca è lunga e non corta come in pseudogagatina. Il margine posteriore del sesto urotergo libero del maschio della nuova specie ha due lobi lunghi, quelli 
di pseudogagatina sono molto corti. L'edeago della nuova specie è ampiamente arcuato al lato ventrale, quello di pseudogagatina rettilineo.

Etimologia: Il nome della nuova specie indica, per la forma dell'edeago e della spermateca, la sua appartenenza a un ramo collaterale ad Atheta pseudogagatina, da quello di questa specie divergente. Prende nome anche dalla Guyana Francese.

\section{Atheta (Datomicra) guyanelegans n. sp. (Figg. 13 e $58-60$ )}

Materiale tipico: Holotype $0^{*}$, Guyane Fr., Caussade, 7.XI.2011, leg. T. Struyve (SDEI).

Paratypes: $2 \sigma^{\top} \sigma^{\top}$, stessa provenienza (IRSNB).

Descrizione: Lunghezza 2,7 mm. Corpo lucido e bruno, capo nero, elitre giallo-brune, uroterghi liberi basali primo e secondo e pigidio rossicci, antenne nero-brune con i due antennomeri basali e base del terzo giallo-rossicci, zampe giallo-rossicce. Occhi più lunghi della regione postoculare, in visione dorsale. Secondo antennomero più corto del primo, terzo più lungo del secondo, quarto a decimo lunghi quanto larghi. Reticolazione di capo ed elitre evidente, quella del pronoto superficiale, quella dell'addome molto trasversa e molto evanescente. Punteggiatura del capo quasi superficiale. Granulosità di pronoto, elitre e addome fine, fitta e saliente, quella del quinto urotergo libero meno fitta. Appiattimento discale del capo. Edeago figg. 58-59, sesto urotergo libero del maschio fig. 60

Comparazioni: La nuova specie per la forma del margine posteriore del sesto urotergo libero del maschio, è simile ad A. conformis (ERICHSON, 1840) della Colombia, di cui ho esaminato la serie tipica (MNHUB). Le spine laterali del margine posteriore del sesto urotergo libero del maschio della nuova specie sono strette e lunghe, quelle di conformis larghe e corte. L'edeago della nuova specie è ampiamente arcuato e sinuoso al lato ventrale, quello di conformis è strettamente arcuato. L'undicesimo antennomero della nuova specie è bruno, quello di conformis giallo-rossiccio.

Etimologia: La nuova specie prende nome dalla forma elegante dell'edeago e dalla Guyana Francese.

\section{Atheta (Datomicra) guydifferens n. sp. (Figg. 14 e 61)}

Materiale tipico: Holotype + , Guyane Fr., Caussade, 7.XI.2011, leg. T. Struyve (IRSNB).
Descrizione: Lunghezza 2,4 mm. Corpo lucido e bruno, quarto urotergo libero nero, antenne brune con i due antennomeri basali e base del terzo giallo-rossicci, zampe giallo-rossicce. Occhi più lunghi della regione postoculare, in visione dorsale. Secondo antennomero più corto del primo, terzo lungo quanto il secondo, quarto a decimo trasversi. Reticolazione di capo ed elitre superficiale, quella del pronoto molto evanescente, quella dell'addome molto trasversa e molto evidente. Punteggiatura del capo fitta e superficiale, assente sulla fascia longitudinale mediana. Granulosità di pronoto ed elitre fitta, fine e saliente. Spermateca fig. 61

Comparazioni: Per la forma della spermateca la nuova specie è simile ad A. alternata (Erichson, 1840) delle Antille, di cui ho esaminato la serie tipica di $10^{\wedge}$ e 4 우 우 (MNHUB). Se ne distingue per il bulbo distale della spermateca meno lungo e con profonda introflessione apicale, mentre è breve in alternata. La parte prossimale della spermateca è corta nella nuova specie, in alternata lunga. La base dell'addome della nuova specie è bruna, quella di alternata giallo-rossiccia.

Etimologia: La nuova specie ha nome che pone l'accento sulla sua differenza da Atheta alternata (ERICHSON, 1840).

\section{Atheta (Datomicra) caussadensis n. sp. \\ (Figg. 15 e 62-65)}

Materiale tipico: Holotype o Guyane Fr., Caussade, 6.XI.2011, leg. T. Struyve (SDEI).

Paratypes: 5 esemplari, stessa provenienza; $10^{\star}$, Guyane Fr., Tonate, 6.XI.2011, leg. T. Struyve (IRSNB).

Descrizione: Lunghezza 1,9 mm. Corpo lucido. Capo, elitre e quarto urotergo libero bruni, pronoto giallo-bruno, base dell'addome e pigidio giallo-rossicci, antenne nere con i due antennomeri basali giallo-rossicci e undicesimo bruno. Occhi più lunghi della regione postoculare, in visione dorsale. Secondo antennomero più corto del primo, terzo lungo quanto il secondo, quarto debolmente trasverso, quinto a decimo trasversi. Reticolazione di capo ed elitre evidente, quella del pronoto superficiale, quella dell'addome molto trasversa ed evidente. Edeago figg. 62-63, spermateca fig. 64, sesto urotergo libero del maschio fig. 65 .

Comparazioni: La spermateca della nuova specie è simile a quella di A. columbica FAUvel, 1901 della Colombia, di cui ho esaminato la serie tipica di $10^{\text {t }}$ e 1 우 (IRSNB). L'introflessione apicale del bulbo distale della spermateca della nuova specie è lunga e stretta, quella di columbica è corta e larga. Ledeago della nuova specie è lungo $0,2 \mathrm{~mm}$, quello di columbica $0,28 \mathrm{~mm}$. L'undice- 
simo antennomero è giallo-rossiccio nella nuova specie, quello di columbica bruno.

Etimologia: La nuova specie prende nome dal toponimo Caussade.

\section{Atheta (Datomicra) camopiensis n. sp. (Figg. 16 e 66)}

Materiale tipico: Holotype + , Guyane Fr., Coralie, 15.XI.2011, leg. T. Struyve (IRSNB).

Descrizione: Lunghezza 2,2 $\mathrm{mm}$. Corpo lucido e nerobruno, elitre brune, antenne nero-brune con i due antennomeri basali bruni, zampe giallo-rossicce. Occhi più lunghi della regione postoculare, in visione dorsale. Secondo antennomero più corto del primo, terzo più lungo del secondo, quarto a decimo trasversi. Reticolazione del capo quasi forte, assente sulla fronte, quella del pronoto superficiale, quella delle elitre evidente, quella dell'addome molto trasversa e quasi forte. Punteggiatura del capo fitta ed evanescente quella delle elitre fitta ed evidente. Granulosità del pronoto fitta e saliente. Spermateca fig. 66.

Comparazioni: Per l'assenza d'introflessione apicale del bulbo distale della spermateca, la nuova specie è comparabile con A. caliginosa BernHAUER, 1934 dell'Argentina, di cui ho esaminato l'olotipo femmina (FMNHC). Il bulbo distale della spermateca della nuova specie è quasi la metà largo quanto quello della spermateca di caliginosa e la sua parte prossimale è più lunga del bulbo distale della stessa spermateca della nuova specie. In caliginosa questa medesima parte prossimale della spermateca è molto più corta della lunghezza del corrispondente bulbo distale della stessa spermateca. Il corpo di caliginosa è giallo-rossiccio con capo e quarto urotergo libero bruno-rossicci, quello della nuova specie è nero con elitre brune.

Etimologia: La nuova specie deriva il suo nome dal fiume Camopi della Guyana Francese.

\section{Atheta (Datomicra) struyvei n. sp. \\ (Figg. 17 e 67-70)}

Materiale tipico: Holotype ${ }^{\star}$, Guyane Fr., Caussade, 7.XI.2011, leg. T. Struyve (IRSNB).

Paratypes: $1 \sigma^{\star}$ e 4 우 우, stessa provenienza; $3 \sigma^{\star} \sigma^{\star}$ e 3 우 우, Guyane Fr., Caussade, 6.XI.2011, leg. T. Struyve (IRSNB, SDEI).

Descrizione: Lunghezza 2,1 mm. Corpo lucido. Capo e quarto urotergo libero neri, pronoto, elitre e terzo urotergo libero bruni, base dell'addome e pigidio gial- lo-rossicci, antenne nere con i due antennomeri basali e base del terzo giallo-rossicci, undicesimo giallo-bruno, zampe giallo-rossicce. Occhi lunghi quanto la regione postoculare, in visione dorsale. Secondo antennomero più corto del primo, terzo più corto del secondo, quarto a decimo trasversi. Reticolazione del capo quasi forte, quella di pronoto ed elitre evidente, quella dell'addome molto trasversa e molto superficiale. Punteggiatura del capo evidente e assente su una stretta fascia longitudinale mediana, quella del pronoto molto fine e fitta. Granulosità di elitre e addome fine e distinta. Edeago figg. 67-68, spermateca fig. 69 , sesto urotergo libero del maschio fig. 70 .

Comparazioni: Per la forma del bulbo prossimale della spermateca, la nuova specie è simile ad A. dichroa (Gravenhorst, 1802) del N America e delle Antille. L'introflessione apicale del bulbo distale della spermateca della nuova specie è corta, mentre è lunga quella di dichroa. L'edeago è lungo $0,22 \mathrm{~mm}$ nella nuova specie, $0,36 \mathrm{~mm}$ nell'olotipo di dichroa (MNHUB). Il pronoto e la base dell'addome della nuova specie sono giallo-rossicci, quelli di dichroa bruni.

Etimologia: La nuova specie è dedicata al suo raccoglitore Tim Struyve, entomologo e consulente di pianificazione di Mechelen, Fiandre, Belgio.

\section{Gyroatheta subg. n. di Atheta THOMson, 1858}

Diagnosi: La forma della spermateca, fig. 73, e i caratteri del sesto urotergo libero del maschio, fig. 74, sono peculiari del genere Gyrophaena Mannerheim, 1831, mai riscontrati nel genere Atheta. La ligula, la formula tarsale 4-5-5 e la struttura dell'edeago si ritrovano nei vari sottogeneri di Atheta, sottogenere Acrotona compresa, e mai in Gyrophaena.

Tipo del nuovo sottogenere Gyroatheta: Atheta (Gyroatheta) guyanicola n. sp.

Etimologia: Il nome del nuovo sottogenere è la fusione dei nomi generici Gyrophaena e Atheta.

\section{Atheta (Gyroatheta) guyanicola n. sp \\ (Figg. 18 e 71-74)}

Materiale tipico: Holotype $\sigma^{\star}$, Guyane Fr., Coralie, 16.XI.2011, leg. T. Struyve (SDEI).

Paratypes: $3 \sigma^{\star} \sigma^{\star}$ e 3 ㅇ 우, stessa provenienza (IRSNB).

Descrizione: Lunghezza 1,6 mm. Corpo lucido e giallo-rossiccio, capo rossiccio, elitre brune, tranne la base giallo-rossiccia, margine posteriore degli uroterghi liberi terzo e quarto bruni, antenne brune con i due 
antennomeri basali gialli, zampe gialle. Occhi lunghi quanto la regione postoculare, in visione dorsale. Secondo antennomero più corto del primo, terzo più corto del secondo, quarto a decimo trasversi. Reticolazione del capo superficiale sul disco, invisibile sul resto della sua superficie, quella di pronoto ed elitre molto evanescente, quella dell'addome visibile alla base del quarto urotergo libero e molto trasversa alla base del quinto. Punteggiatura del capo fine, fitta e assente sulla fascia longitudinale mediana. Granulosità di pronoto ed elitre fitta e molto superficiale. Edeago figg. 71-72, spermateca fig. 73, sesto urotergo libero del maschio fig. 74 .

Etimologia: La nuova specie ha nome che significa "abitatrice della Guyana Francese".

\section{Lamprostiba pulchra n. sp.}

(Figg. 19 e 75)

Materiale tipico: Holotype + , Guyane Fr., Caussade, 7.XI.2011, leg. T. Struyve (IRSNB).

Descrizione: Lunghezza $1,6 \mathrm{~mm}$. Corpo lucido e giallo-rossiccio, capo rossiccio, elitre, tranne la base, e quarto urotergo libero bruni, antenne brune con i tre antennomeri basali gialli e l'undicesimo giallo-bruno, zampe giallo-rossicce. Occhi più lunghi della regione postoculare, in visione dorsale. Secondo antennomero più corto del primo, terzo più corto del secondo, quarto a decimo trasversi. Reticolazione del corpo superficiale, quella degli uroterghi liberi quarto e quinto molto trasversa $\mathrm{e}$ pure superficiale. Punteggiatura del capo fitta e assente sulla fascia longitudinale mediana. Granulosità del pronoto fitta ed evanescente, quella delle elitre fitta e saliente. Spermateca fig. 75.

Comparazioni: La nuova specie è distinta da L. schwabei (Bernhauer, 1939) del Cile, di cui ho esaminato la serie tipica (FMNHC), per la forma della spermateca e colore del corpo. La parte prossimale della spermateca della nuova specie è molto arcuata, mentre in schwabei lo è debolmente. Il corpo di schwabei è bruno, quello della nuova specie giallo-rossiccio con capo rossiccio ed elitre, tranne la base, e quarto urotergo libero bruni.

Etimologia: La nuova specie è chiamata "bella" per il colore vario del corpo.

\section{Elixusa subg. n. di Parademosoma Bernhauer, 1929}

Diagnosi: Per la forma dell'edeago e la punteggiatura sparsa del pronoto, il nuovo sottogenere è distinto da Parademosoma (Parademosoma). La forma della ligula divisa in due larghi lobi non permette di istituire un nuovo genere. Essa è uguale che in Parademosoma. I caratteri sottogenerici sono i seguenti:
1. Parte prossimale dell'edeago prolungata in una o più spire; pronoto sparsamente punteggiato Elixusa subg. n. di Parademosoma

Parte prossimale dell'edeago semplice, non come sopra; pronoto fittamente punteggiato Parademosoma (Parademosoma)

Tipo del sottogenere Elixusa: Parademosoma (Elixusa subg. n.) guyanensis n. sp.

Etimologia: Il nome femminile del nuovo sottogenere deriva dal greco antico $\dot{\varepsilon} \lambda_{1} \xi=$ spira e oú $\sigma \alpha=$ che è. La spira è quella basale dell'edeago.

\section{Parademosoma (Elixusa) guyanensis n. sp. (Figg. 20 e 76-77)}

Materiale tipico: Holotype $\sigma^{\star}$, Guyane Fr., Caussade, 7.XI.2011, leg. T. Struyve (SDEI).

Paratype: $1 \sigma^{\star}$, stessa provenienza (IRSNB).

Descrizione: Lunghezza 2,1 mm. Corpo lucido e bruno, capo nero, elitre giallo-brune, antenne brune con i due antennomeri basali giallo-rossicci, zampe giallo-rossicce. Occhi più lunghi della regione postoculare, in visione dorsale. Secondo antennomero più corto del primo, terzo più corto del secondo, quarto a decimo trasversi. Reticolazione del capo evidente, quella del resto del corpo invisibile. Punteggiatura del capo fitta, evanescente e assente sulla fronte. Punteggiatura del pronoto superficiale e sparsa, quella delle elitre fitta ed evanescente. Granulosità dell'addome poco fitta e saliente. Edeago figg. 76-77.

Comparazioni: Al nuovo sottogenere Elixusa appartiene sicuramente anche P. venezuelana PACE, 2011 del Venezuela, per la forma dell'edeago. Per la forma dell'edeago la nuova specie è simile a essa. La parte prossimale dell'edeago della nuova specie è avvolta in una sola spira, quella di venezuelana avvolta in sei spire. Il pronoto della nuova specie è trasverso, quello di venezuelana appena trasverso.

Etimologia: La nuova specie prende nome dalla Guyana Francese.

\section{Dolerella n. gen.}

Diagnosi: Habitus simile a Myllaena ErICHSON, 1837, ma le parti orali e la formula tarsale 4-5-5 differenti. Per l'habitus e la formula tarsale uguale, come per la forma della spermateca, il nuovo genere è simile al genere Dinusella Bernhauer, 1908 del Sudamerica, di cui ho esaminato il tipo del genere Dinusella brasi- 
liana Bernhauer, 1908 del Brasile (FMNHC). Il nuovo genere se ne distingue per i caratteri dati nella seguente chiave:

1. Corpo tozzo, mesocoxe separate, ligula divisa in due lobi Dinusella BernHAUER

- Corpo slanciato, simile a Myllaena, mesocoxe contigue, ligula intera, fig. 81 ........ Dolerella n. gen.

Tipo del nuovo genere: Dolerella cayennensis n. sp.

Etimologia: Il nome femminile del nuovo genere signi-

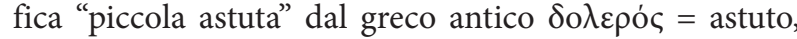
ingannevole. È, infatti, facile cadere nell'inganno di attribuire la specie a Myllaena.

\section{Dolerella cayennensis n. sp.}

(Figg. 21 e 78-83)

Materiale tipico: Holotype $\sigma^{\star}$, Guyane Fr., Coralie, 16.XI.2011, leg. T. Struyve (SDEI).

Paratypes: 26 esemplari, stessa provenienza (IRSNB, SDEI).

Descrizione: Lunghezza $1,8 \mathrm{~mm}$. Corpo poco lucido e bruno, pronoto e base dell'addome giallo-bruni, antenne brune con i due antennomeri basali gialli, zampe gialle. Occhi lunghi quanto la regione postoculare, in visione dorsale. Secondo antennomero più lungo del primo, terzo più corto del secondo, quarto a decimo trasversi. L'intero corpo coperto di fitta pubescenza sericea e fine su un fondo granuloso. Edeago figg. 78-79, spermateca fig. 80 .

Etimologia: La nuova specie è dedicata alla capitale della Guyana Francese, Cayenne.

\section{Dinusella angularis $\mathrm{n}$. sp.}

(Figg. 22 e 84-86)

Materiale tipico: Holotype $\sigma^{\star}$, Guyane Fr., Caussade, 7.XI.2011, leg. T. Struyve (IRSNB).

Paratypes: 7 esemplari, stessa provenienza; $10^{*}$, Guyane Fr., Coralie, 15.XI.2011, leg. T. Struyve (IRSNB, SDEI).

Descrizione: Lunghezza 2,4 mm. Corpo lucido e giallo-rossiccio, capo bruno-rossiccio, elitre brune con angoli posteriori esterni giallo-rossicci, quarto urotergo libero e base del quinto bruni, antenne e zampe giallo-rossicce. Occhi più corti della regione postoculare, in visione dorsale. Secondo antennomero lungo quanto il primo, terzo più lungo del secondo, quarto a ottavo più lunghi che larghi, nono lungo quanto largo, decimo trasverso. Reticolazione del corpo non visibile. Punteggiatura del capo fine, fitta e quasi invisibile. Granulosità del pronoto fitta e molto superficiale, quella delle elitre fitta e saliente, quella dell'addome evidente. Edeago figg. 84-85, spermateca fig. 86 .

Comparazioni: La spermateca della nuova specie è simile a quella di D. brasiliana Bernhauer, 1908, di cui ho esaminato la serie tipica (FMNHC). Se ne distingue per la parte prossimale della spermateca avvolta in numerose e strette spire, mentre in brasiliana la parte prossimale della spermateca descrive ampi meandri aggrovigliati. L'edeago della nuova specie è completamente differente da quello di brasiliana, con apice, in visione ventrale, a punta di lancia, mentre in brasiliana l'apice è ogivale.

Etimologia: La nuova specie prende nome dalla presenza degli angoli posteriori delle elitre giallo-rossicci.

\section{Dinusella pulchripennis n. sp.}

(Figg. 23 e 87)

Materiale tipico: Holotype + , Guyane Fr., Caussade, 6.XI.2011, leg. T. Struyve (SDEI).

Paratypes: 2 우, Guyane Fr., Tonate, 6.XI.2011, leg. T. Struyve (IRSNB).

Descrizione: Lunghezza $3 \mathrm{~mm}$. Corpo lucido e giallo-rossiccio, elitre giallo-rossicce con una fascia postomerale bruna estesa fino all'angolo posteriore interno, quarto urotergo libero bruno, antenne e zampe giallo-rossicce. Occhi lunghi quanto la regione postoculare, in visione dorsale. Secondo antennomero lungo quanto il primo, terzo più lungo del secondo, quarto a ottavo più lunghi che larghi, ottavo lungo quanto largo, decimo trasverso. Reticolazione del corpo invisibile. Punteggiatura del capo molto fine, fitta ed evidente. Granulosità del pronoto molto fitta e superficiale, quella delle elitre fitta e poco saliente, quella dell'addome fitta ed evidente. Spermateca fig. 87.

Comparazioni: La spermateca della nuova specie è simile a quella di D. ornata PACE, 1990 dell'Argentina e Cile. Se ne distingue per il differente colore delle elitre. Quelle di ornata non hanno macchie giallo-rossicce come nella nuova specie. Il bulbo prossimale della spermateca della nuova specie è più largo di quello di ornata e la parte distale della stessa spermateca è ampiamente ricurva, mentre è strettamente arcuata in ornata.

Etimologia: La nuova specie ha nome che significa "belle elitre". 


\section{Ringraziamenti}

Per avermi affidato in studio il materiale oggetto del presente lavoro rivolgo i miei più cordiali ringraziamenti al collega e naturalista belga Tim Struyve di Mechelen. Per il prestito di tipi ringrazio il Dr. A. Newton del Field Museum of Natural History di Chicago e il Dr. Manfred Uhlig del Museum für Naturkunde der HumboldtUniversität, Berlino.

\section{Bibliografia}

Bernhauer, M. 1908: Beitrag zur Staphylinidenfauna von Südamerika. - Archiv für Naturgeschichte 74: 283-372.

Bernhauer, M. 1927: Zur Staphylinidenfauna Südamerikas, insbesondere Argentiniens. - Archiv für Naturgeschichte 91: 229-264.

Bernhauer, M. 1929: Neue Staphyliniden aus Mittelamerika. - Wiener Entomologische Zeitung 46: 186-208.

Bernhauer, M. 1934: Neue Staphyliniden aus Argentiniens. - Revista de Entomologia do Rio de Janeiro 3: 501-517.

Bernhauer, M. 1939: New Staphyliniden aus Chile. Arbeiten über morphologische und taxonomische Entomologie 6: 12-15.

Bernhauer, M. \& Scheerpeltz, O. 1926: Staphylinidae VI. - In: Schenkling, 3. ed., Coleopterorum Catalogus, Pars 82: 499-988. - W. Junk, Berlin.

Erichson, W. F. 1837: Die Käfer der Mark Brandenburg Vol. I, Abt. 1. 384 pp. - Berlin.

ERICHSON, W. F. 1840: Genera et species staphylinorum insectorum coleopterorum familiae - F. H. Morin, Berlin.

Fauvel, A. 1901: Voyage de M. le Dr. Ed. Bugnion au Venezuela, en Colombie et aux Antilles. - Revue d'Entomologie 20: 69-91.

Gravenhorst, J. L. C. 1802: Coleoptera microptera Brunsvicensia nec non exoticorum quotquot exstant in collectionibus entomologorum Brunsvicensium in genera, familias et species distribuit. - Brunsuigae: Carolus Reichard, LXIV + 206 pp.
Mannerheim, C. G. 1830: Précis d'un nouvel arrangement de la Famille des Brachélytres de l'Ordre des Insectes Coléoptères. - Mémoires de l'Académie des Sciences de St. Petersbourg 1 : 415-501.

Mulsant, M. E. \& Rey, C. 1873: Description de divers Coléoptères Brévipennes nouveaux ou peu connus. Opuscula Entomologica 15: 147-189.

PACE, R. 1987: Aleocharinae dell'isola della Guadalupa (Antille) (Coleoptera: Staphylinidae). - Folia Entomologica Hungarica 48: 187-205.

PACE, R. 1990a: Nuovi Falagriini, Hoplandriini ed Aleocharini della Regione Neotropica (Coleoptera, Staphylinidae) (LXXX Contributo alla conoscenza delle Aleocharinae). - Giornale italiano d'Entomologia 5: 157-180.

PACE, R. 1990b: Aleocharinae neotropiche del Museo Ungherese di Storia Naturale (Coleóptera, Staphylinidae). - Annales Historico-Naturales Musei Nationalis Hungarici 81: 53-107.

PACE, R. 1996: Aleocharinae della Colombia e dell' Ecuador. Parte II. (Coleóptera, Staphylinidae). Revue suisse de Zoologie 103: 643-676.

PACE, R. 2008: New records of Aleocharinae from Ecuador and Peru, with the description of new species, new subgenera and new genera (Coleoptera, Staphylinidae) pp. 225-398. - In: Giachino, P. M. (ed.) 2008: Biodiversity of South America I. Memoirs on Biodiversity, World Biodiversity Association onlus, Verona, 1: $496 \mathrm{pp}$.

PACE, R. 2011: New and additional records, new genera and new species of Aleocharinae from Venezuela, Ecuador and Peru (Coleoptera, Staphylinidae). Bollettino del Museo Civico di Storia Naturale di Verona, Botanica Zoologia 35: 43-70.

SAwADA, K. 1980: Atheta and its allies of Southeast Asia (Coleoptera: Staphylinidae). I. Reexamination of some species from Borneo and Singapore. - Pacific Insects 21: 335-354.

Thomson, C. G. 1858: Försök till uppställning af Sveriges Staphyliner. - Öfversigt af Kongliga VetenskapsAkademiens Förhandlinger 15: 27-40. 


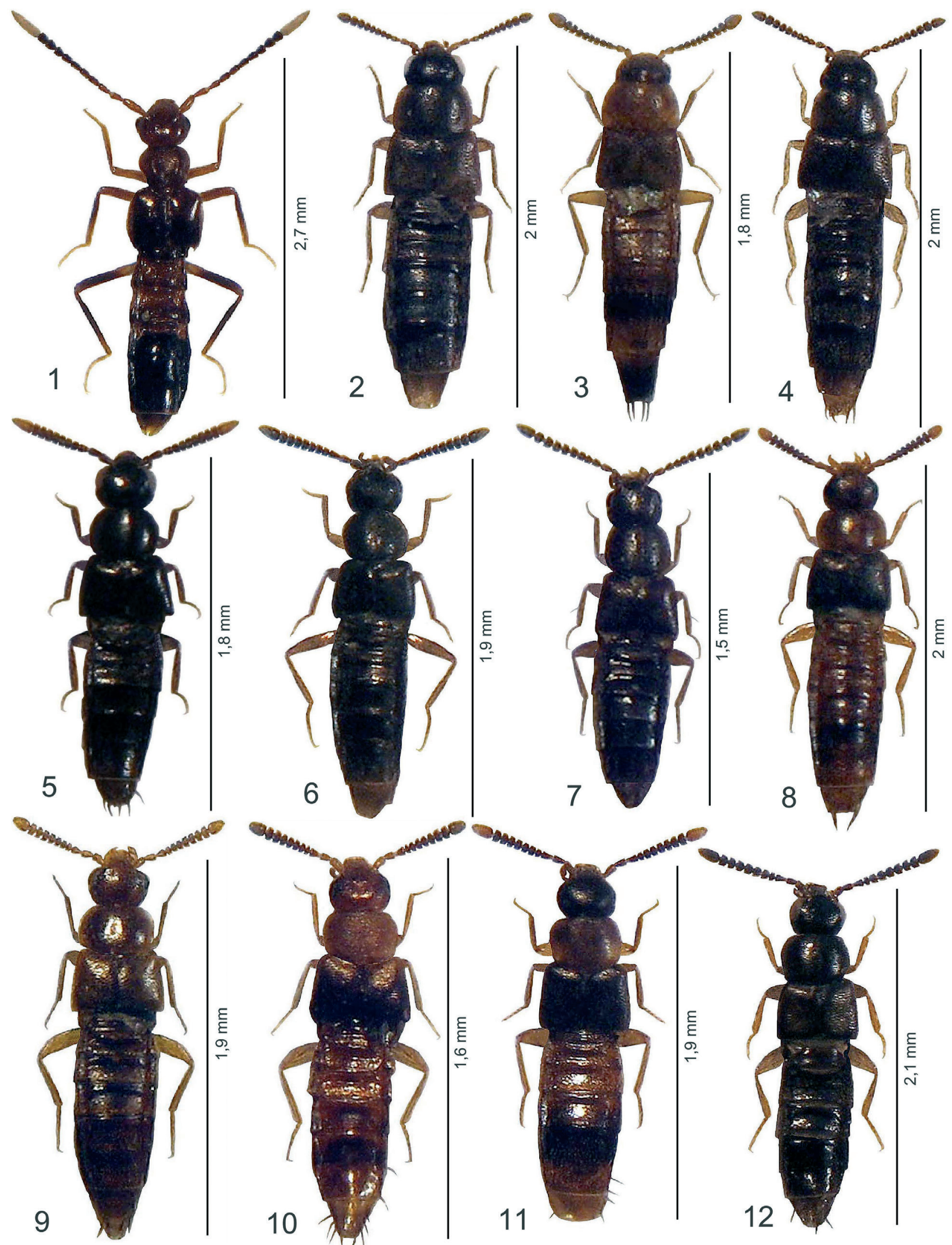

Figg. 1-12: Habitus. 1. Ischnopoda pulchritudinis n. sp.; 2. Xyronarchusa amazonica n. gen., n. sp.; 3. Atheta (Acrotona) obscurepyga n. sp.; 4. Atheta (Acrotona) tanpokensis n. sp.; 5. Atheta (Acrotona) guyterminicornis n. sp.; 6. Atheta (Acrotona) subparcior n. sp.; 7. Atheta (Acrotona) guyminima n. sp.; 8. Atheta (Microdota) ungulifera n. sp.; 9. Atheta (Microdota) guyarufa n. sp.; 10. Atheta (Xestota) guyattenuata n. sp.; 11. Atheta (Datomicra) guymimetica n. sp.; 12. Atheta (Datomicra) guydivergens n. sp. 


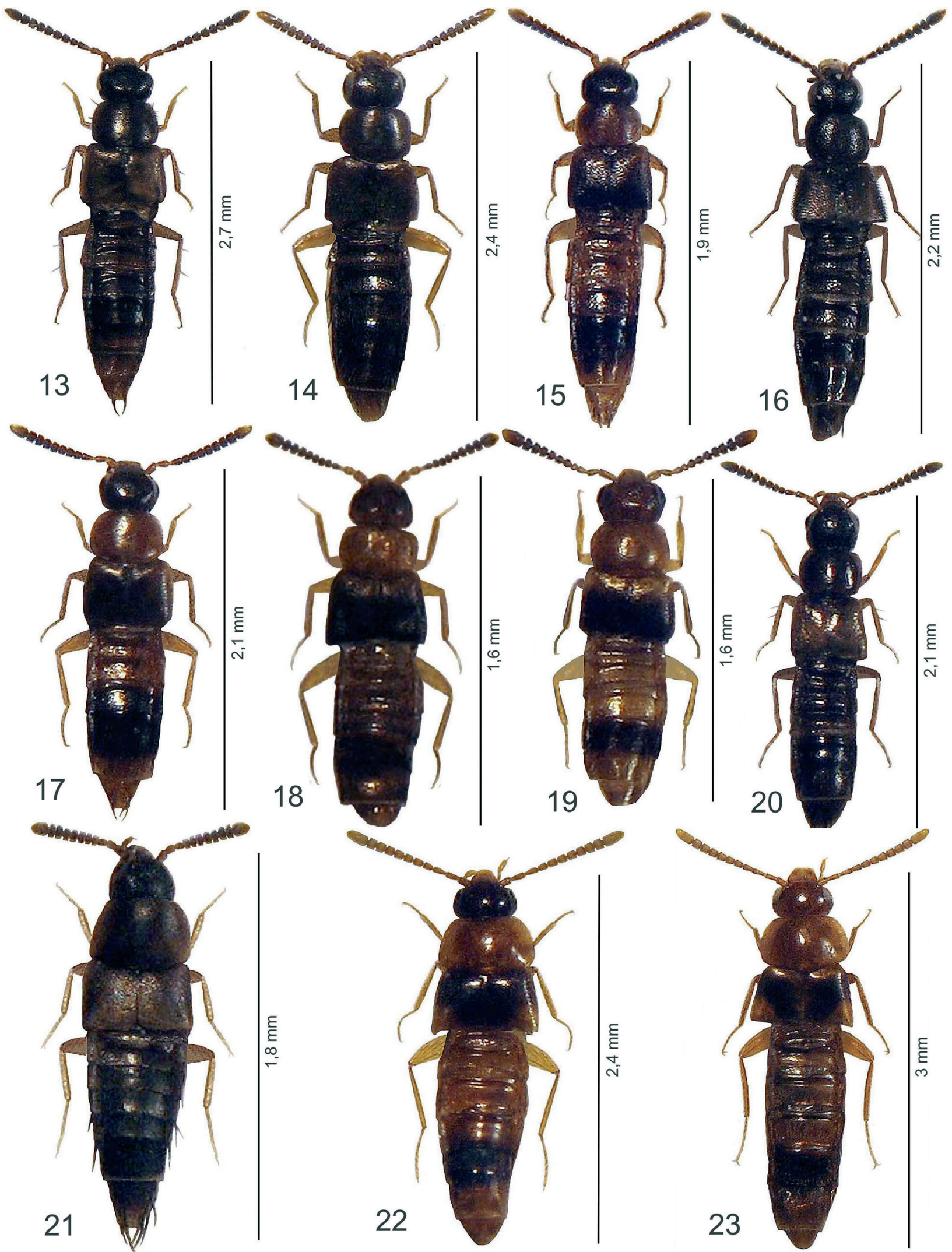

Figg. 13-23: Habitus. 13. Atheta (Datomicra) guyanelegans n. sp.; 14. Atheta (Datomicra) guydifferens n. sp.; 15. Atheta (Datomicra) caussadensis n. sp.; 16. Atheta (Datomicra) camopiensis n. sp.; 17. Atheta (Datomicra) struyvei n. sp.; 18. Atheta (Gyroatheta subg. n.) guyanicola n. sp.; 19. Lamprostiba pulchra n. sp.; 20. Parademosoma (Elixusa subg. n.) guyanensis n. sp.; 21. Dolerella cayennensis n. sp.; 22. Dinusella angularis n. sp.; 23. Dinusella pulchripennis $\mathrm{n}$. sp. 

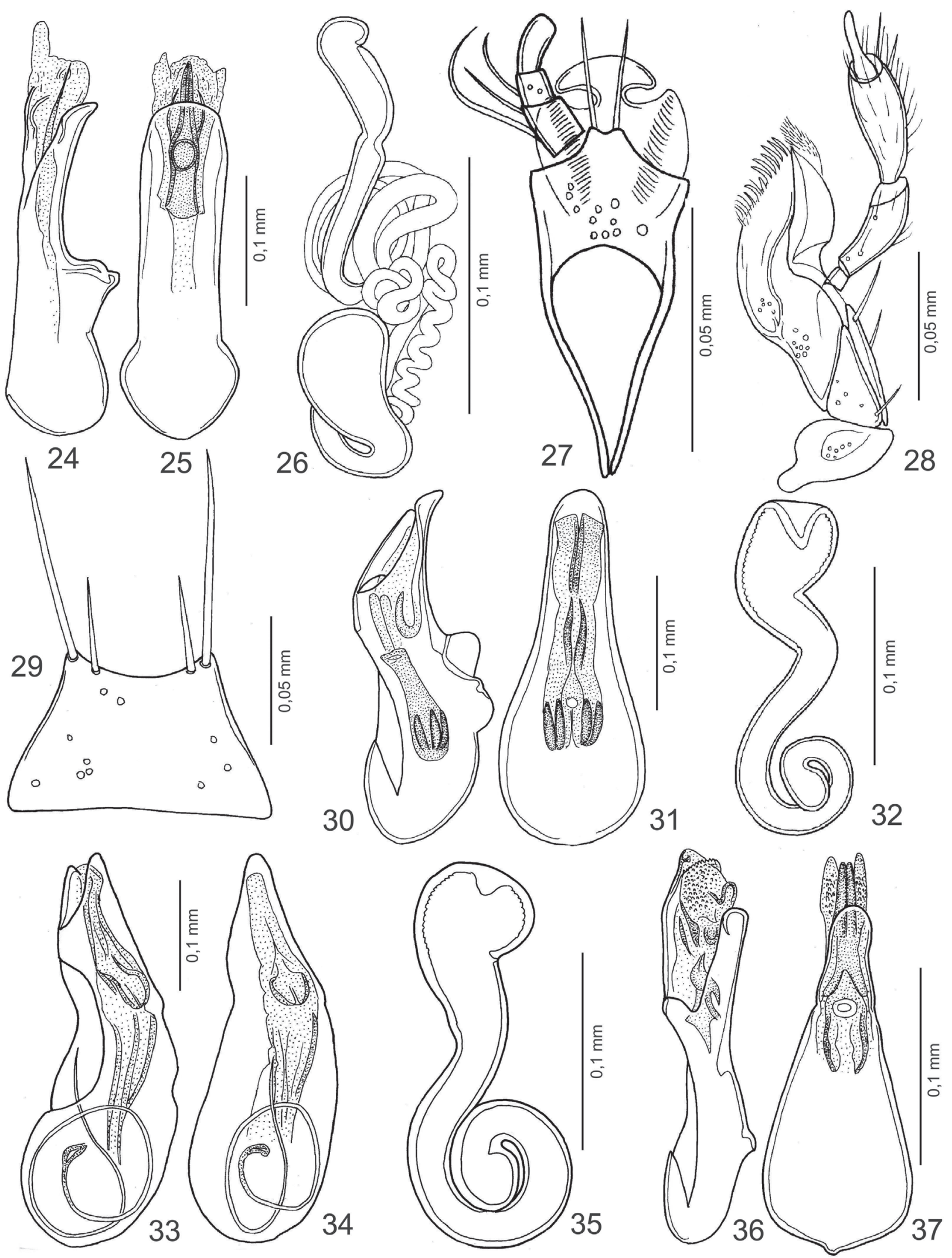

Figg. 24-37: Edeago in visione laterale e ventrale, spermateca, labio con palpo labiale, maxilla con palpo maxillare e mento. 24-25. Ischnopoda pulchritudinis n. sp.; 26-29. Xyronarchusa amazonica n. gen., n. sp.; 30-32. Atheta (Acrotona) obscurepyga n. sp.; 33-35. Atheta (Acrotona) tanpokensis n. sp.; 36-37. Atheta (Acrotona) guyterminicornis n. sp. 


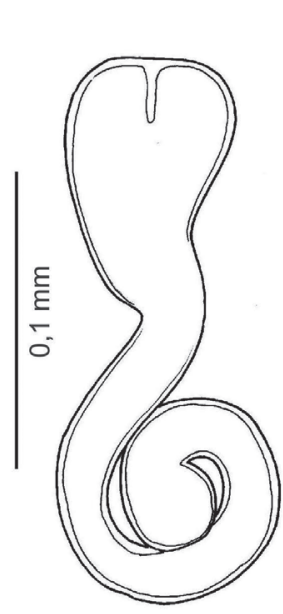

38

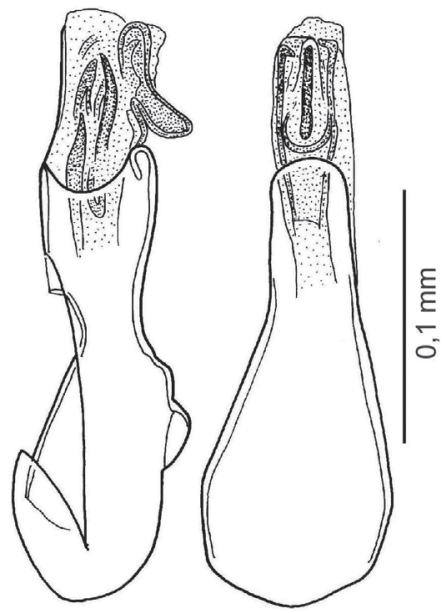

39

40

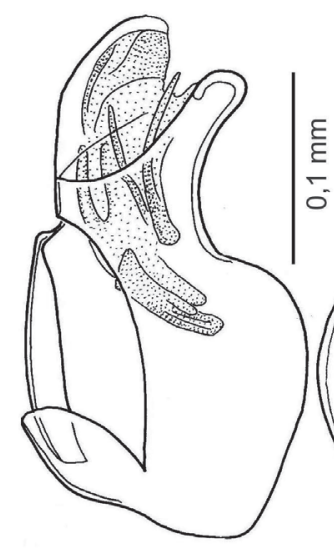

46

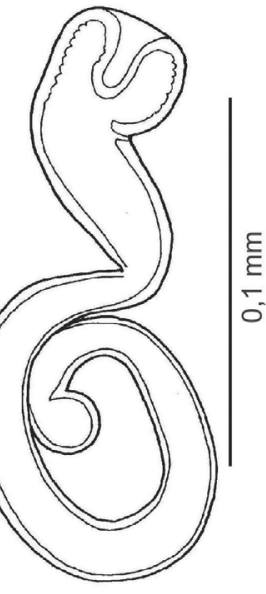

41

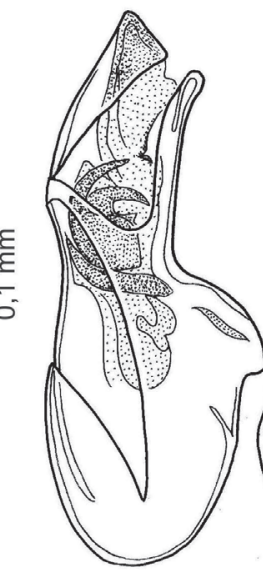

42

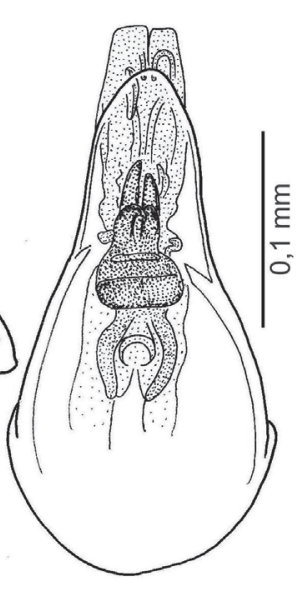

43

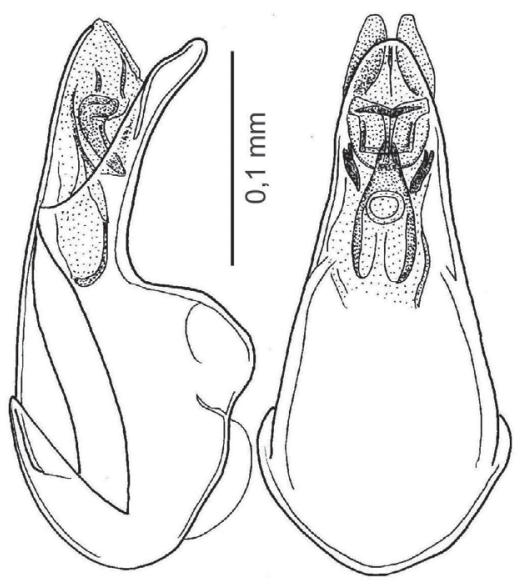

44

45

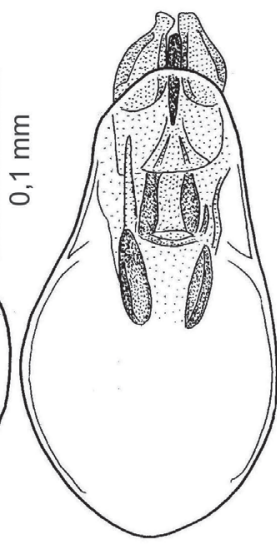

47

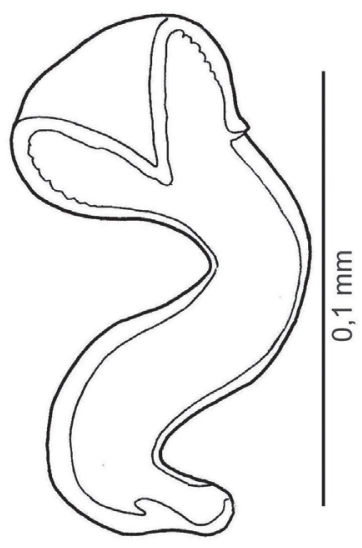

48

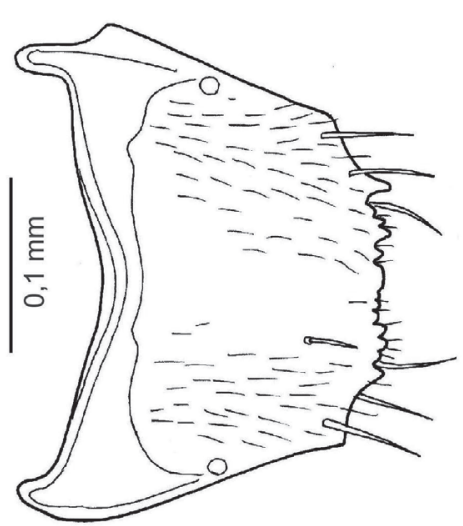

49

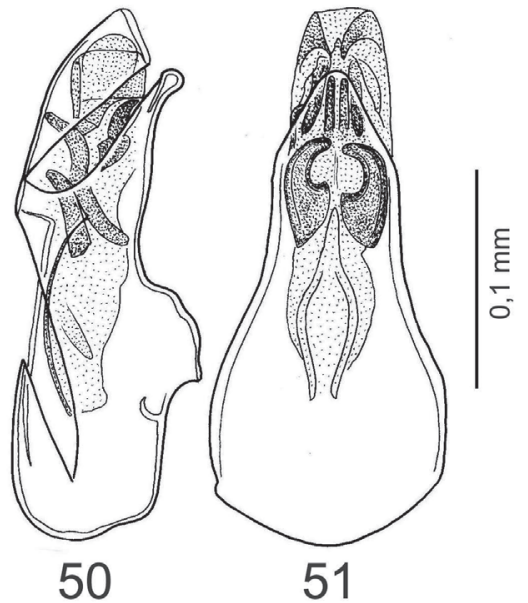

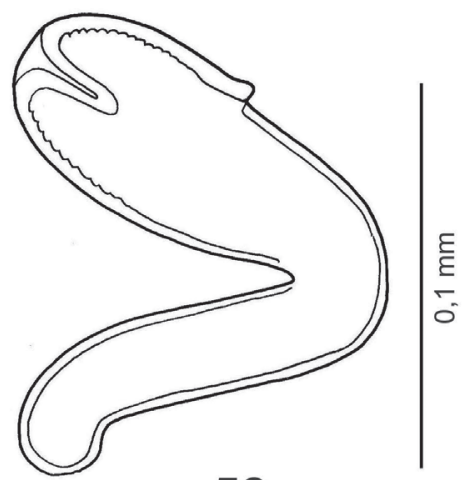

52

Figg. 38-52: Spermateca, edeago in visione laterale e ventrale e sesto urotergo libero del maschio. 38. Atheta (Acrotona) subparcior n. sp.; 39-41. Atheta (Acrotona) guyminima n. sp.; 42-43. Atheta (Microdota) ungulifera n. sp.; 44-45. Atheta (Microdota) guyarufa n. sp.; 46-49. Atheta (Xestota) guyattenuata n. sp.; 50-52. Atheta (Datomicra) guymimetica n. sp. 

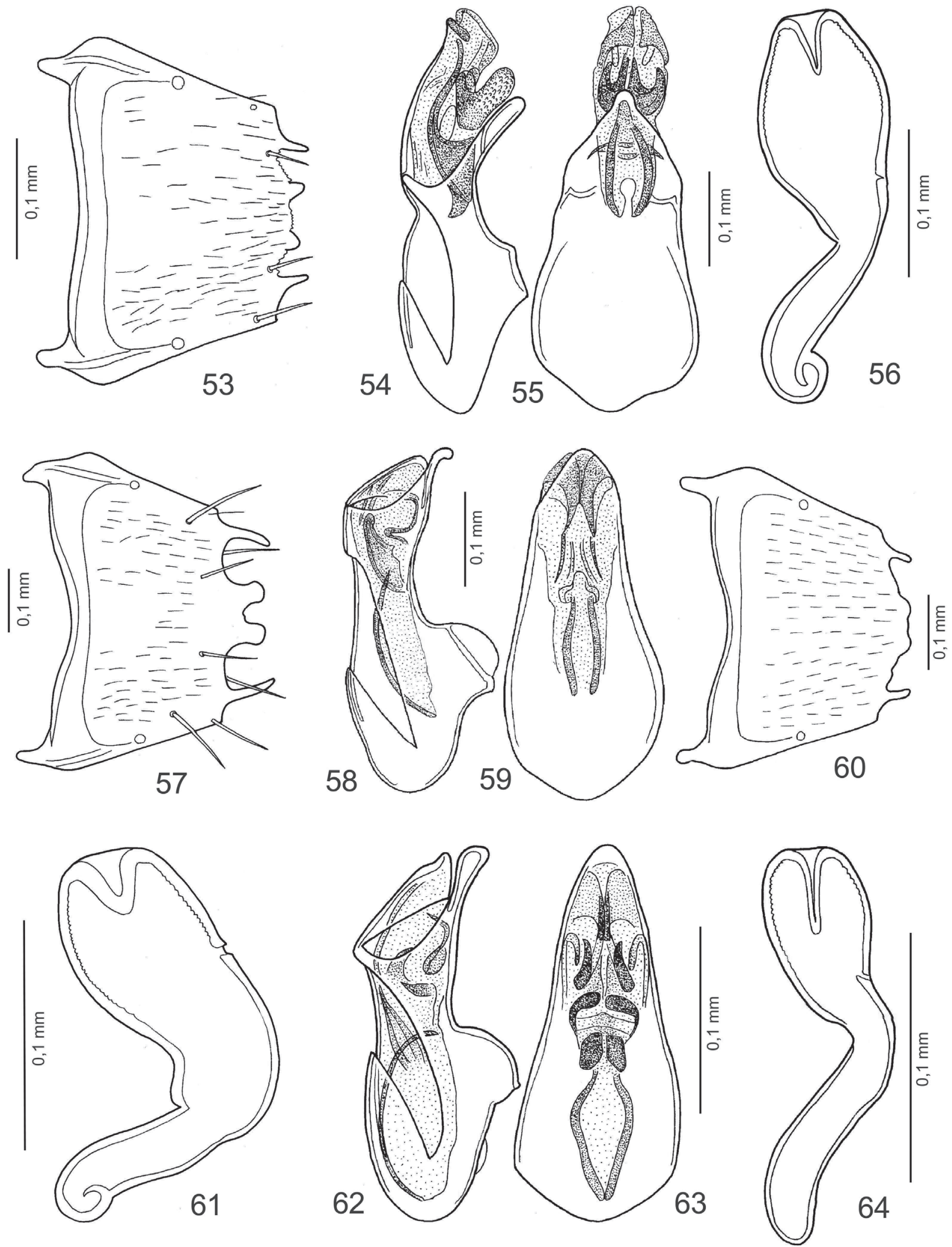

Figg. 53-64: Sesto urotergo libero del maschio, edeago in visione laterale e ventrale e spermateca. 53. Atheta (Datomicra) guymimetica n. sp.; 54-57. Atheta (Datomicra) guydivergens n. sp.; 58-60. Atheta (Datomicra) guyanelegans n. sp.; 61. Atheta (Datomicra) guydifferens n. sp.; 62-64. Atheta (Datomicra) caussadensis n. sp. 

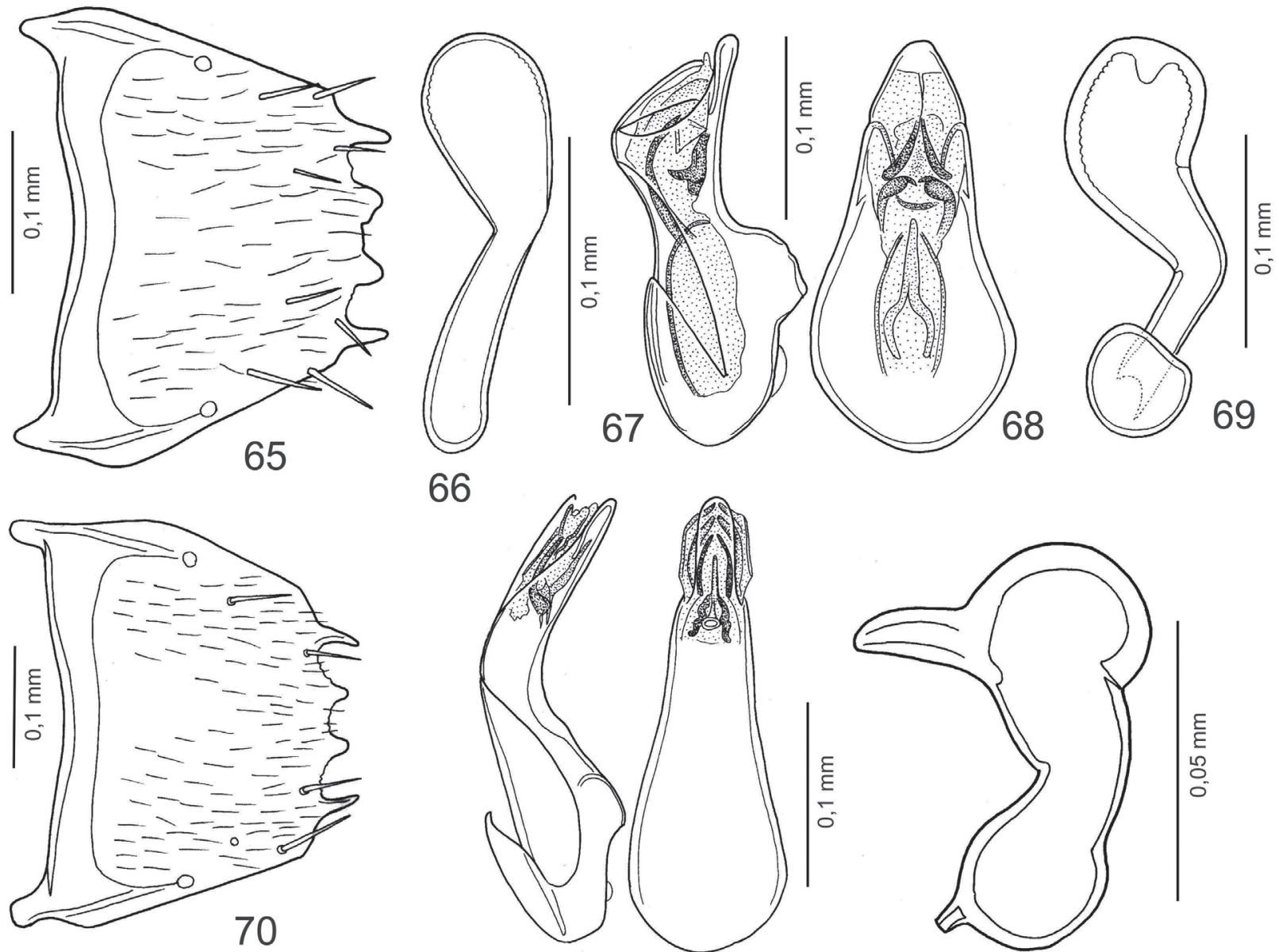

66
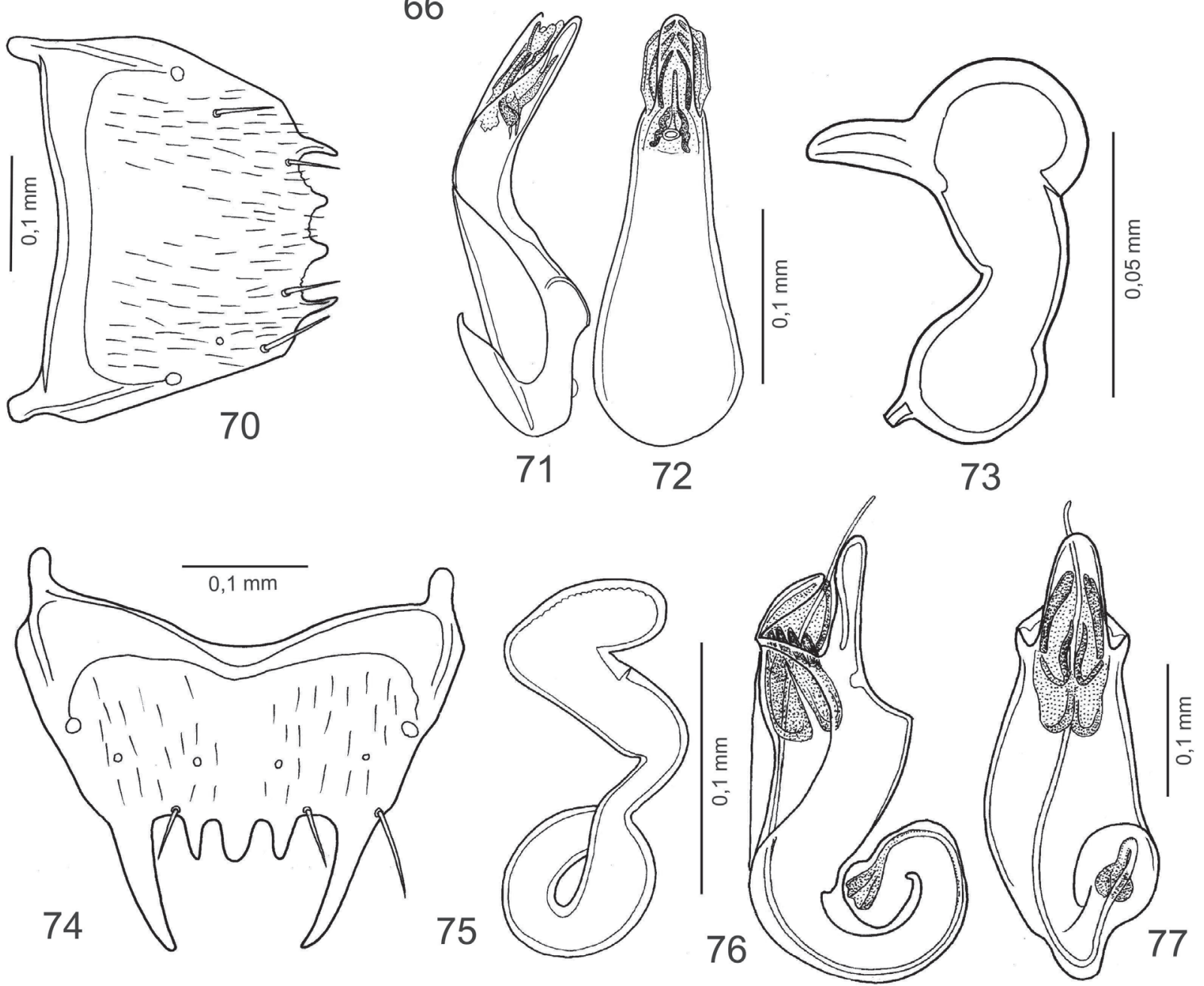

Figg. 65-77: Sesto urotergo libero del maschio, spermateca, edeago in visione laterale e ventrale. 65. Atheta (Datomicra) caussadensis n. sp.; 66. Atheta (Datomicra) camopiensis n. sp.; 67-70. Atheta (Datomicra) struyvei n. sp.; 71-74. Atheta (Gyroatheta subg. n.) guyanicola n. sp.; 75. Lamprostiba pulchra n. sp.; 76-77. Parademosoma (Elixusa subg. n.) guyanensis n. sp. 

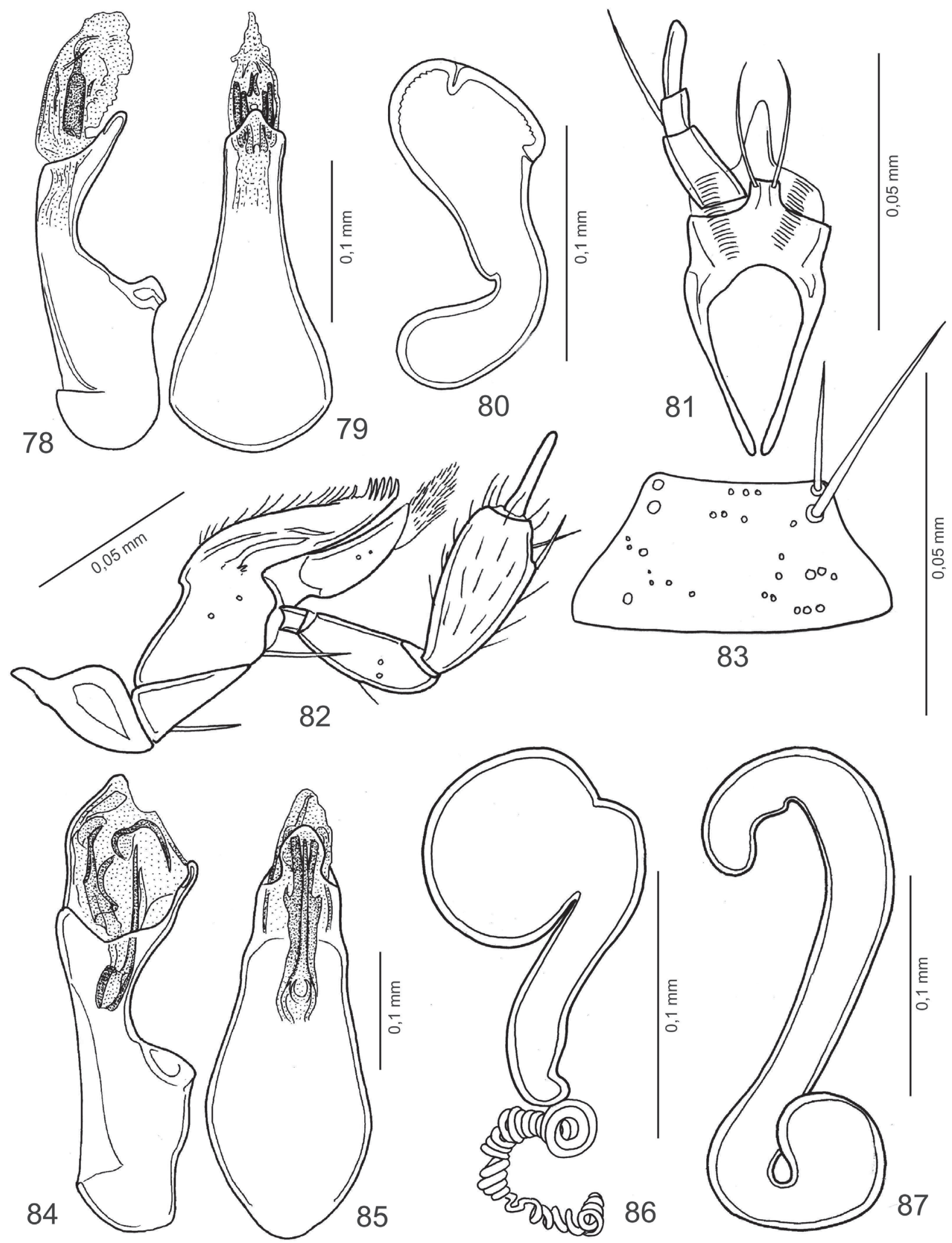

Figg. 78-87: Edeago in visione laterale e ventrale, spermateca, labio con palpo labiale, maxilla con palpo maxillare e mento. 78-83. Dolerella cayennensis n. sp.; 84-86. Dinusella angularis n. sp.; 87. Dinusella pulchripennis $\mathrm{n}$. $\mathrm{sp}$. 